\title{
Multi-Tb/s Photonic Transceivers for Metro Optical Network Connectivity Evolution
}

\author{
M. Svaluto Moreolo*a , L. Nadal ${ }^{\mathrm{a}}$, J. M. Fabrega ${ }^{\mathrm{a}}$, F. J. Vilchez ${ }^{\mathrm{a}}$, Christian Neumeyr $^{\mathrm{b}}$, \\ Alberto Gatto ${ }^{c}$, Paola Parolaric, Pierpaolo Boffic \\ ${ }^{a}$ Centre Tecnològic de Telecomunicacions de Catalunya (CTTC/CERCA), Av. C. F. Gauss 7, 08860 \\ Castelldefels (Barcelona), Spain; ' Vertilas GmbH, Daimlerstr. 11d, D-85748 Garching, Germany; \\ ${ }^{\mathrm{c}}$ Politecnico di Milano, P.zza Leonardo da Vinci 32, 20133, Milano, Italy
}

\begin{abstract}
Metro area network (MAN) connectivity is rapidly evolving towards a much more dense, complex and diverse scenario to be dynamically addressed with flexible cost-efficient and high-capacity technology and architecture solutions, dealing with an even more open and disaggregated paradigm. In this work, sliceable bandwidth/bitrate variable transceiver (SBVT) architectures adopting modular approach and suitable photonic technologies (such as VCSEL), enabling to efficiently and dynamically exploit both spectral and spatial dimensions, are discussed, considering design, implementation, cost and flexibility aspects. Recent numerical and experimental results are reported, showing how to enable scalability towards supporting multi-Tb/s connectivity in flexible and dynamic large MAN.
\end{abstract}

Keywords: Metro optical networks, sliceable bandwidth/bitrate variable transceivers (S-BVT), disaggregated optical networks, advanced photonic transceiver architectures, programmable transceivers, software defined networking.

\section{INTRODUCTION}

Metro area network (MAN) connectivity is rapidly evolving towards a much more dense, complex and diverse scenario, to give support to a myriad of new services and applications, while dynamically adapt to the need for huge capacity/speed as well as high quality of service and instant data access ${ }^{1}$. This translates in a set of challenging requirements to be satisfied with novel and improved technologies/architectures.

The design of programmable photonic transceivers targeting multi- $\mathrm{Tb} / \mathrm{s}$ speed assumes a key role, particularly if sliceable with adaptive bandwidth/bitrate functionalities. On one hand, the target is providing flexibility, programmability and interoperability in the context of an even more open and disaggregated paradigm ${ }^{2}$. On the other hand, a reduction of cost, power consumption and footprint should be addressed, exploring and exploiting cost-effective photonic devices and photonic integration ${ }^{3,4,5}$. Alternative system solutions can be proposed adopting either direct or external modulation, combined with direct detection (DD) or coherent receiver (CO-Rx) ${ }^{6}$. Adopting multicarrier modulation (MCM) at the digital signal processing (DSP) allows a fine spectral granularity and manipulation, thanks to the ability of accessing and suitably loading the electrical subcarriers. In particular, combining orthogonal frequency division multiplexing (OFDM) with optical single sideband (SSB) filtering allows improving the spectral efficiency and extending the achievable reach ${ }^{7,8}$. Adopting a modular approach to aggregate multiple flows in the spectral dimension enhances the capacity exploiting the frequency dimension, while enabling super-granularity. Indeed, the adoption of multiple bands allows further improving the capacity scale. On this regard, multi-band technology allows to enhance the capacity by exploiting the entire low loss single mode fiber (SMF) spectrum, ranging from $1260 \mathrm{~nm}$ up to $1620 \mathrm{~nm}^{9}$. Finally, with polarization division multiplexing (PDM) and spatial division multiplexing (SDM), the supported capacity is improved by a factor of $2 M$, where the double of the capacity derives from PDM and $M$ is the number of fibers/cores and/or modes used in case of adopting fiber bundles or multicore fibers (MCF) and/or multimode (few-mode) fibers ${ }^{5,10}$,

${ }^{11}$. In this paper, transceiver architectures allowing an optimal network resources usage, including the exploitation of multiple dimensions, for enabling future connectivity towards supporting multi- $\mathrm{Tb} / \mathrm{s}$ will be presented.

*michela.svaluto@cttc.es; phone+34 9364529 00; www.cttc.es 
In particular, sliceable bandwidth/bitrate variable transceiver (S-BVT) architectures for the metro/aggregation network segment, also suitable for distributed data center interconnection, will be described. We report recent numerical and experimental results, considering design, implementation, cost and flexibility aspects. Also, the implementation and assessment in a real testbed network will be presented. Cost-effective solutions adopting a modular approach and exploiting spectrum and space dimensions will be discussed, showing how to enable up to $50 \mathrm{~Gb} / \mathrm{s}$ per flow and scalability towards multi- $\mathrm{Tb} / \mathrm{s}$ capacity in a grow-as-needed fashion. The use of vertical cavity surface emitting lasers (VCSELs) will be particularly analyzed as promising option for supporting the connectivity evolution in flexible and dynamic large MAN.

\section{PHOTONIC TRANSCEIVERS}

\subsection{S-BVT architectures}

Programmable S-BVTs become key enablers of high-speed optical transmission by the exploitation of different dimensions such as spectrum, polarization and space. Wavelength division multiplexing (WDM) can be implemented by transmitting multiple signals/slices at different wavelengths. Specifically, different slices can be enabled according to the traffic demand and network condition following a pay-as-you grow model ${ }^{12}$. Additionally, PDM allows doubling the spectral efficiency (SE), as well as including an additional dimension that can be also used for routing additional traffic in dense fiber links as shown in $^{13}$. SDM allows scaling the capacity of a factor depending on the adopted fibers or cores of a multicore fiber $(\mathrm{MCF})^{5,11}$.

Figure 1 shows different architecture options to design and implement a programmable modular system adopting alternative S-BVT architectures.

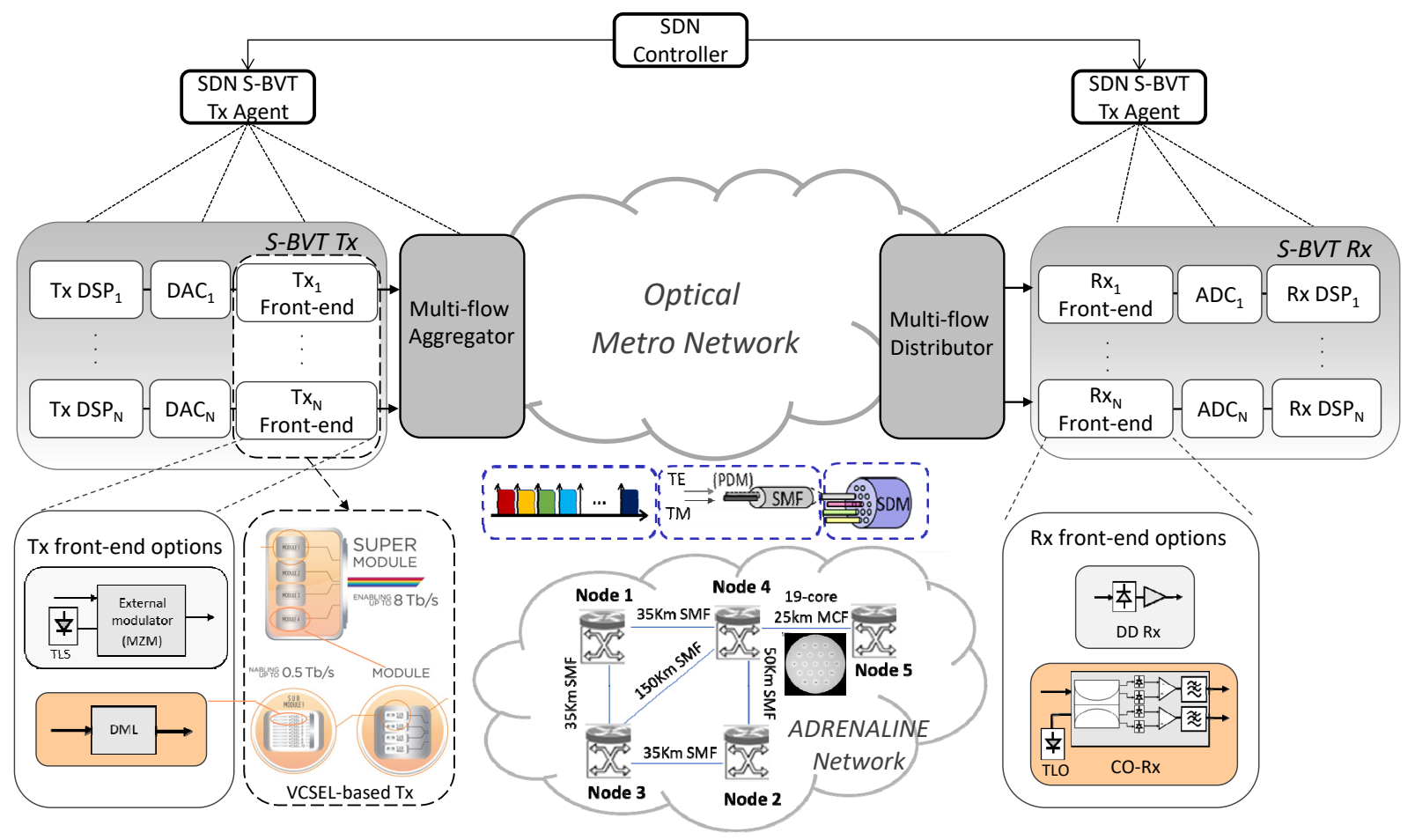

Figure 1. Schematic of the programmable S-BVT system. In the insets: Tx and Rx front-end options, modular integrated VCSEL-based S-BVT Tx. Spectral, polarization and spatial resources; ADRENALINE testbed network with 19-core MCF.

The modular architecture of the S-BVT provides variable capacity, scalable with the number of enabled slices enhancing network flexibility and dynamicity. A software defined networking (SDN) controller activates the suitable slices/flows 
according to the available resources, appropriately configuring the hardware/devices composing the S-BVT and the network nodes for establishing a successful connection within the metro network ${ }^{2,4,5,7,12}$.

As shown in Figure 1 insets, different design options are possible for the optoelectronic front-ends at the S-BVT transmitter (Tx) and receiver (Rx). Direct modulation (DM) of an array of direct modulated lasers (DMLs) or external modulation of tuneable laser sources (TLSs) are alternative solutions at the Tx, trading-off cost-effectiveness and flexibility. DD or CO-Rx are possible solutions to adopt at the Rx side, trading-off low-cost and ultimate performance in terms of reach and transmission robustness. In this work, we focus on design solutions based on external modulation combined with DD and direct modulation with CO-Rx, in order to target the metro network segment needs and requirements in terms of cost, capacity/reach and flexibility. In fact, adopting external modulation with TLS has higher cost compared to the use of DML, in particular when VCSEL sources are adopted ${ }^{4}$, while DD allows to compensate this cost at the Rx side compared with the adoption of a CO-Rx. DM with DD is the most cost-efficient solution, at the expenses of the achievable reach/performance. MCM, either discrete multitone (DMT) or OFDM, are considered for targeting a flexible supported capacity up to $50 \mathrm{~Gb} / \mathrm{s}$ per each single flow at the S-BVT. Adaptive bit and power loading at the DSP provides rate adaptation and capacity/performance maximization. Multi-Tb/s can be achieved enabling multiple flows and multiple dimensions, fully exploiting the available metro network resources. This includes covering the entire C-band (and beyond, if multiple bands transmission is envisioned/enabled according to the designed architecture) and adopting SDM and/or PDM. The S-BVT design should allow achieving this goal with high flexibility/dynamicity at reduced cost and optimized resource usage to efficiently target the metro network requirements.

\subsection{S-BVT based on external modulation and DD}

The S-BVT building block at the Tx consists of an external Mach Zehnder modulator (MZM) and a TLS. Different slices/flows at different wavelengths can be created by suitably tuning the TLS of each S-BVT Tx building block. Intensity modulation (IM) is performed at the Tx front-end. In order to improve the spectral efficiency and achievable reach, SSB filtering is performed at the multi-flow aggregator, either implemented as a network element included in the S-BVT design or within the network node. At the Rx side, a simple photodiode with a transimpedance amplifier (TIA) is used. In order to further improve the system/network capacity, polarization and space can also be exploited. Two OFDM signals/slices generated with this (S)-BVT configuration can be multiplexed into orthogonal polarization states (PDM), enabling 50\% spectral saving and promoting resource dynamicity ${ }^{13}$. SDM is enabled by transmitting the different slices over multiple fibers in a bundle or multiple cores of a MCF, or even few mode fibers, in order to overcome the physical limits of today's SMF-based systems.

\subsection{VCSEL-based S-BVT}

The S-BVT building block at the Tx adopts a DM VCSEL at a certain operating wavelength. Short cavity (SC) VCSEL technology offers high modulation bandwidth $(>18 \mathrm{GHz})$ but limited tuneability with respect to adopting a Tx front-end solution including a tuneable laser source ${ }^{8}$. Within the EU H2020 PASSION project ${ }^{14}$, a photonic integrated modular VCSEL-based Tx is considered to target the metro network needs in terms of flexibility and cost. Each flow enables to support up to $50 \mathrm{~Gb} / \mathrm{s}$ thanks to the adoption of adaptive DSP with MCM and SC-VCSEL technology. As shown in Figure 1, the fundamental Tx module consists of 40 VCSELs integrated in a single photonic chip at operating wavelengths covering the C-band. So that, to select the operating wavelength for a specific connection, the appropriate VCSEL element should be activated ${ }^{4}$. At the Rx, either DD or CO-Rx can be adopted. Indeed, the CO-Rx offers ultimate performance and increasing flexibility adopting a tunable local oscillator (TLO) $)^{5}$. It is worth noting that a simplified coherent reception is required when combined with DM at the Tx side ${ }^{5,8}$.

\section{ASSESSING METRO NETWORK CONNECTIVITY}

To assess the support of metro network connectivity adopting the S-BVT architectures described in Section 2, we evaluated the performance in terms of capacity/reach of a single flow of the S-BVT. The experimental assessment of the different design options has been performed in the ADRENALINE testbed ${ }^{15}$, either using the photonic mesh network depicted in Figure 1 and/or multiple standard SMF spools, adopting wavelength selective switches (WSS) and arrayed waveguide gratings (AWGs) as multiplexer/demultiplexers for implementing the cascading nodes. As shown in Figure 1, the ADRENALINE testbed network consists of 4 nodes (optical cross-connects, OXCs, and reconfigurable add-drop multiplexers, ROADMs) with amplified SMF links of $35 \mathrm{~km}, 50 \mathrm{~km}$ and $150 \mathrm{~km}$, and also includes a 19-core MCF with fan-in and fan-out towards node 5 . 
At the DSP block of the S-BVT Tx, a randomly generated data stream is mapped into different constellations such as BPSK and M-QAM (with $M=2 n$ and $2 \leq n \leq 8$ ) according to the channel profile. A bit and power loading (BL/PL) algorithm (e.g., Levin Campello) is implemented to optimize the modulation format and power value per MCM (OFDM) subcarrier according to the channel profile ${ }^{12}$. The channel state information is retrieved adopting uniform loading (4QAM) ${ }^{6}$. The overheads due to cyclic prefix (CP) and training symbols (TS) are $1.9 \%$ and $4 \%$, respectively. A hard decision (HD) forward error correction (FEC) of $7 \%$, corresponding to a bit error rate (BER) of $4.62 \cdot 10^{-3}$, is considered. The BER is calculated by error counting. The digital real-valued OFDM signal (with $20 \mathrm{GHz}$ or $16 \mathrm{GHz}$ bandwidth) is converted to analogue by using a digital-to-analogue converter (DAC) at $64 \mathrm{GSa} / \mathrm{s}$. The analogue-to-digital conversion (ADC) is performed, for the experimental assessment, using a digital oscilloscope with sample rate up to $100 \mathrm{GSa} / \mathrm{s}$.

In case of adopting MZM and TLS at the S-BVT Tx building block, IM is performed to be combined with DD at the Rx. At the multi-flow aggregator, optical SSB is performed. Considering a $20 \mathrm{GHz}$ bandwidth signal, the optical bandwidth occupation in a flex-grid network is of two frequency slots of $12.5 \mathrm{GHz}(25 \mathrm{GHz})$. The maximum capacity achieved in back-to-back (B2B) adopting this configuration is greater than $60 \mathrm{~Gb} / \mathrm{s}$ (up to $70 \mathrm{~Gb} / \mathrm{s}$ ) ${ }^{2}, 11$, thus above the considered target capacity per flow of $50 \mathrm{~Gb} / \mathrm{s}$.

We performed the experimental assessment of this configuration considering spectral, polarization and spatial resources. Results are reported in Table 1. It is shown that $40 \mathrm{~Gb} / \mathrm{s}$ and $30 \mathrm{~Gb} / \mathrm{s}$ per slice/flow can be achieved after traversing 100 $\mathrm{km}$ and $200 \mathrm{~km}$ of standard SMF (SSMF), respectively ${ }^{12}$. Thus, aggregating multiple (m) flows with m=25 provides a total capacity of $1 \mathrm{~Tb} / \mathrm{s}$ or $750 \mathrm{~Gb} / \mathrm{s}$, respectively. Considering, the transmission over 10 cascading nodes, including 100GHz AWGs and 50-GHz WSS, and the $120 \mathrm{~km}$ 3-hop path (2 amplified links of $35 \mathrm{~km}$ and 1 link of $50 \mathrm{~km})$ of the ADRENALINE network, $35 \mathrm{~Gb} / \mathrm{s}$ capacity per slice can be transmitted, according to ${ }^{2}$. When the granularity at the WSS nodes is $25 \mathrm{GHz}$, the capacity decreases to $30 \mathrm{~Gb} / \mathrm{s}$. By implementing PDM, an aggregated capacity of $82 \mathrm{~Gb} / \mathrm{s}$ is obtained after transmitting over 1-hop $35 \mathrm{~km}$ path of the ADRENALINE testbed. In particular, $44 \mathrm{~Gb} / \mathrm{s}$ and $38 \mathrm{~Gb} / \mathrm{s}$ can be transmitted in $\mathrm{H}$ and $\mathrm{V}$ polarization components, at $33.5 \mathrm{~dB}$ OSNR, ensuring the target BER ${ }^{13}$. However, at the increase of the optical link length, the polarization mode dispersion (PMD) effect appears, which causes the misalignment of the state of polarization (SOP) between the OFDM subcarriers and the optical carrier resulting in power fading. Finally, to explore the spatial dimension, the experimental assessment over the 19-core MCF path of $25.4 \mathrm{~km}$ of the ADRENALINE testbed has been performed in presence of crosstalk (XT) effect, by filling the adjacent cores with OFDM signals. A capacity per slice/core of $54 \mathrm{~Gb} / \mathrm{s}$ is obtained at the target BER activating the adjacent cores (while 57 $\mathrm{Gb} / \mathrm{s}$ is achievable without XT) with $31.6 \mathrm{~dB}$ OSNR ${ }^{11}$. This capacity can be scaled up with the number of cores enabling $\mathrm{Tb} / \mathrm{s}$ transmission (above $1 \mathrm{~Tb} / \mathrm{s}$ with 19 cores MCF). Considering a recirculating loop including $11 \mathrm{spans}$ of $25.4 \mathrm{~km}$ $(279 \mathrm{~km}) 19$-core $\mathrm{MCF}$, the data rate decreases to $24 \mathrm{~Gb} / \mathrm{s}^{11}$. It is important to note that XT effect and nonlinearities degrade the performance and reduce the achievable capacity per flow. Specifically, for an input power of $6 \mathrm{dBm}$, the nonlinear effects start dominating the transmission after $220 \mathrm{~km}$; reach at which the achievable capacity is still above 30 $\mathrm{Gb} / \mathrm{s}$ (with and without XT) ${ }^{12}$.

Table 1 also reports the results in case of adopting VCSEL-based S-BVT. When SC-VCSEL technology is adopted, the modulation bandwidth can be higher than $18 \mathrm{GHz}$, however, the VCSEL adopted in the experiments has a bandwidth limited to $10 \mathrm{GHz}$, mainly due to the device packaging. Indeed, the use of MCM modulation, with adaptive bandwidth up to $16 \mathrm{GHz}$, and applying pre-emphasis at the Tx DSP, allow to more efficiently fill the $25 \mathrm{GHz}$ optical channel. This allows obtaining a maximum capacity of $36.9 \mathrm{~Gb} / \mathrm{s}$ in the $\mathrm{B} 2 \mathrm{~B}$ case with $\mathrm{DD}^{16}$. We have verified that adopting an integrated CO-Rx module similar performance as adopting DD can be achieved in B2B. Particularly, at the OSNR value of $33.4 \mathrm{~dB}$, using a commercial integrated $\mathrm{CO}-\mathrm{Rx}$, a capacity of $34.6 \mathrm{~Gb} / \mathrm{s}$ is supported close to the value supported by DD $(32.8 \mathrm{~Gb} / \mathrm{s})$ and higher than the one obtained by adopting a discrete CO-Rx with similar responsivity $(27.6 \mathrm{~Gb} / \mathrm{s})^{16}$. Similar performance is achieved after a 2-hop $15 \mathrm{~km}$ path, as reported in Table 1 . The chromatic dispersion and chirp effects severely degrade the performance over longer path, when combining DM-VCSEL with DD, particularly when double sideband DMT is adopted. Thanks to the adoption of SSB filtering and CO-Rx, large MAN connectivity can be supported $^{5,8}$. The connectivity over multi-hop paths including multiple nodes with different functionalities has been experimentally analysed in the ADRENALINE testbed network ${ }^{16}$. In particular, $22.6 \mathrm{~Gb} / \mathrm{s}$ capacity per VCSEL flow has been demonstrated over up to 6-hop $160 \mathrm{~km}$ path, including $135 \mathrm{~km}$ of amplified SMF links and $25 \mathrm{~km} \mathrm{MCF}{ }^{16}$. This result is obtained using a CO-Rx with discrete component. Similar to the case of external modulation with DD, we have analysed the performance of a VCSEL-based S-BVT adopting integrated CO-Rx, considering the spatial resource. Specifically, we have analysed the case with or without XT of the adjacent cores for the 19-core MCF path (25.4 km) between ADRENALINE node-4 and node-5. 
Table 1. Performance of different S-BVT configurations for different scenarios set-up in the ADRENALINE testbed. BW indicates the bandwidth of the electrical/digital MCM (OFDM) signal adaptively modulating the Tx front-end. The VCSEL bandwidth is $10 \mathrm{GHz}$. The considered FEC threshold for all cases is $4.62 \times 10^{-3}$. (a) Case with 10 cascading nodes including $100-\mathrm{GHz}$ AWGs and 50-GHz WSS; (b)aggregated data rate including $44 \mathrm{~Gb} / \mathrm{s}$ (H polarization component) and $38 \mathrm{~Gb} / \mathrm{s}$ (V polarization component); ${ }^{(\mathrm{c})}$ Recirculating loop considering multiple spans of $25.4 \mathrm{~km}$ of 19-core MCF.

\begin{tabular}{|c|c|c|c|c|c|}
\hline $\begin{array}{l}\text { S-BVT Tx } \\
\text { front-end }\end{array}$ & $\begin{array}{l}\text { S-BVT Rx } \\
\text { front-end }\end{array}$ & $\begin{array}{c}\text { BW } \\
(\mathrm{GHz})\end{array}$ & $\begin{array}{c}\text { Capacity } \\
(\mathbf{G b} / \mathbf{s})\end{array}$ & $\begin{array}{c}\text { Length } \\
\text { (km) }\end{array}$ & Scenario $^{\text {Ref }}$ \\
\hline MZM\&TLS & $\mathrm{DD}$ & 20 & 50 & 35 & 1-hop path ${ }^{2}$ \\
\hline MZM\&TLS & $\mathrm{DD}$ & 20 & 40 & 100 & SSMF link $^{12}$ \\
\hline MZM\&TLS & $\mathrm{DD}$ & 20 & 35 & 120 & $\begin{array}{l}\text { Multi-path }^{2} \\
10 \text { cascading nodes }^{(a)}\end{array}$ \\
\hline MZM\&TLS & DD & 20 & 30 & 200 & SSMF link ${ }^{12}$ \\
\hline MZM\&TLS & $\mathrm{DD}$ & 20 & $82^{\text {(b) }}$ & 35 & 1-hop path \& PDM $^{13}$ \\
\hline MZM\&TLS & $\mathrm{DD}$ & 20 & 54 & 25.4 & MCF path ${ }^{11}$ \\
\hline MZM\&TLS & DD & 20 & 24 & $279.4^{(\mathrm{c})}$ & MCF loop ${ }^{11}$ \\
\hline DM SC-VCSEL & $\mathrm{DD}$ & 16 & 33.7 & 15 & 2-hop path ${ }^{16}$ \\
\hline DM SC-VCSEL & $\begin{array}{l}\mathrm{CO}-\mathrm{Rx} \\
\text { (Discrete) }\end{array}$ & 16 & 26.4 & 15 & 2-hop path ${ }^{16}$ \\
\hline DM SC-VCSEL & DD & 16 & 7.6 & 160 & 6-hop path \& $\mathrm{MCF}^{16}$ \\
\hline DM SC-VCSEL & $\begin{array}{l}\text { CO-Rx } \\
\text { (Discrete) }\end{array}$ & 16 & 22.6 & 160 & 6-hop path \& $\mathrm{MCF}^{16}$ \\
\hline DM SC-VCSEL & $\begin{array}{l}\text { CO-Rx } \\
\text { (Integrated) }\end{array}$ & 16 & 24.5 & 25.4 & MCF path w/o XT \\
\hline DM SC-VCSEL & $\begin{array}{l}\text { CO-Rx } \\
\text { (Integrated) }\end{array}$ & 16 & 23.4 & 25.4 & MCF path with XT \\
\hline
\end{tabular}

The capacity supported by a single flow of the VCSEL-based S-BVT is $24.5 \mathrm{~Gb} / \mathrm{s}$ without (w/o) XT. Activating the adjacent cores, the capacity (with XT) at similar OSNR value slightly decreases to $23.4 \mathrm{~Gb} / \mathrm{s}$. We have obtained some preliminary results also in case of implementing a loop to assess the robustness of the transmission over a longer MCF path targeting large MAN connectivity. With the increase of MCF length and due to appearance of nonlinearities, as well as the OSNR value reduction (also due to the loop implementation), the capacity decreases. Specifically, we have found that after $50.8 \mathrm{~km}(2 \times 25.4 \mathrm{~km})$ the capacity is $20.5 \mathrm{~Gb} / \mathrm{s}$, after $101.6 \mathrm{~km}$ is $17.8 \mathrm{~Gb} / \mathrm{s}$ and after more than $200 \mathrm{~km}$ the capacity results halved with respect to the value after $25.4 \mathrm{~km}$.

When considering higher bandwidth SC-VCSELs, $50 \mathrm{~Gb} / \mathrm{s}$ capacity per flow can be targeted even with this S-BVT configuration, as numerically demonstrated $\mathrm{in}^{5}$. Particularly, considering $18 \mathrm{GHz} \mathrm{VCSEL}$ and integrated CO-Rx, the supported capacity per flow over the $160 \mathrm{~km}$ 6-hop path is higher than $40 \mathrm{~Gb} / \mathrm{s}$. Thus, $1.6 \mathrm{~Tb} / \mathrm{s}$ can be supported activating all the spectral flows of a fundamental VCSEL-based S-BVT integrated module. Furthermore, $50 \mathrm{~Gb} / \mathrm{s}$ per flow can be supported over $200 \mathrm{~km}$ path if an OSNR above $30 \mathrm{~dB}$ can be ensured, as experimentally demonstrated in ${ }^{17}$. When considering additional spectral flows and fine granularity $(25 \mathrm{GHz})$ exploiting also the spatial dimension, multi$\mathrm{Tb} / \mathrm{s}$ can be supported with the proposed S-BVT architecture. 


\subsection{Results discussion and design guidelines}

MZM \& TLS enhanced flexibility and performance at the expenses of the Tx cost. Thus, it is convenient to adopt DD for a cost-effective S-BVT implementation. Adaptive OFDM-based DSP and SSB filtering allows enhancing the solution flexibility and covering the metro network segment. Multi-Tb/s capacity is achieved by aggregating multiple spectral flows and exploiting the spatial dimension, introducing an additional degree of freedom in the architecture design. PDM allows doubling the spectral efficiency and achievable capacity, however at the increase of the optical link length, the PMD effect appears resulting in power fading.

VCSEL technology is emerging as efficient (in terms of cost, power consumption and footprint) alternative to external modulation in metro networks. The spectral efficiency is improved by adopting MCM, pre-emphasis and SSB optical filtering. SC-VCSEL with high bandwidth modulation allows to improve the capacity performance to target $50 \mathrm{~Gb} / \mathrm{s}$ per flow. Photonic integrated VCSEL-based S-BVT Tx with modular composition enables to cost-effectively face the capacity demand of MAN networks. Specifically, with the design envisioned and implemented in the framework of the EU H2020 PASSION project ${ }^{14}$, up to $2 \mathrm{~Tb} / \mathrm{s}$ can be achieved with a photonic integrated chip module enabling up to 40 VCSEL flows covering the C-band ${ }^{5}$. Even if the SC-VCSEL tunability is limited, the flexibility is enabled at the SDN controller by suitably enabling the VCSEL sources with the appropriate operating wavelengths. In order to cover the expected reach in large MAN, this S-BVT Tx configuration is conveniently combined with CO-Rx at the receiver side.

Both the proposed configurations imply an optical bandwidth occupation per flow of two frequency slots of $12.5 \mathrm{GHz}$, (minimum channel/flow spacing of $25 \mathrm{GHz}$ ). When adopting $25 \mathrm{GHz}$ granularity at the network nodes, the filter narrowing effect has a greater impact on the capacity performance with respect to the case of $50 \mathrm{GHz}$, and this should be taken into account in the architecture design and number of required slices/flows to be activated ${ }^{2,5}$. However, a reduction on the modulated bandwidth, even if decreasing the spectral efficiency, could be beneficial for reducing the adjacent spectral channel crosstalk 5 .

If MCF is adopted (instead of using a bundle of fibers for SDM), crosstalk effect and nonlinearities should be taken into account, since they have an impact on the expected performance, reducing the maximum supported capacity per flow. Indeed, this depends on the target reach and would be more critical for the connectivity within large MANs.

\section{CONCLUSIONS}

Transceiver architectures allowing an optimal network resources usage, including the exploitation of multiple dimensions, for enabling future connectivity, have been presented. S-BVT architectures for the metro/aggregation network segment have been described, considering alternative implementation options and different aspects. Recent results have been reported also including experimental assessment performed in a real testbed network (CTTC ADRENALINE testbed). Cost-effective solutions adopting a modular approach and exploiting spectrum and space dimensions have been discussed, showing how to enable up to $50 \mathrm{~Gb} / \mathrm{s}$ per flow and scalability towards multi- $\mathrm{Tb} / \mathrm{s}$ capacity in a grow-as-needed fashion. The use of VCSELs has been also analyzed as promising option for supporting the connectivity evolution in flexible and dynamic large MAN.

\section{ACKNOWLEDGMENT}

This paper is part of a project that has received funding from the European Union's Horizon 2020 research and innovation programme under grant agreement PASSION No 780326. This work has been also partially supported by the Spanish AURORAS (RTI2018-099178-B-I00) project.

\section{REFERENCES}

[1] CISCO White Paper: "Cisco Visual Networking Index: Forecast and Trends, 2017-2022”, (2019).

[2] Nadal, L., et al., "SDN-enabled Sliceable Transceivers in Disaggregated Optical Networks", in J. Lightwave Technol. (2019).

[3] Nadal, L., et al., "Programmable SDN-enabled S-BVT based on hybrid electro-optical MCM", IEEE/OSA J. Opt. Commun. Netw., 10, 593-602 (2018). 
[4] Svaluto Moreolo, M., Fabrega, J. M., Nadal, L., Martínez, R., Casellas, R., "Synergy of Photonic Technologies and Software-Defined Networking in the Hyperconnectivity Era," IEEE/OSA J. Lightwave Technol., 37, 16, $3902-3910$ (2019).

[5] Svaluto Moreolo, M., et al., "Programmable VCSEL-based photonic system architecture for future agile Tb/s metro networks," J. Optical Commun. and Networking, OFC 2020 Special Issue, Vol. 13, No. 2, pp. A187A199 (2021).

[6] Svaluto Moreolo, M., et al., "Sliceable BVT Evolution Towards Programmable Multi-Tb/s Networking," Electronics 2019, 8, 1476 (2019).

[7] Svaluto Moreolo, M., et al. "SDN-Enabled Sliceable BVT Based on Multicarrier Technology for Multiflow Rate/Distance and Grid Adaptation," IEEE/OSA J. Lightwave Technol., 34, 1516-1522 (2016).

[8] Svaluto Moreolo, M., Nadal, L., Fabrega, J. M., Vilchez, F. J., Neumeyr, C., Gatto, A., Parolari, P., Boffi, P., "VCSEL-based sliceable bandwidth/bitrate variable transceivers," Proc. SPIE 10946, Metro and Data Center Optical Networks and Short-Reach Links II, 1094606 (2019), DOI: 10.1117/12.2509316.

[9] Napoli, A., et al., "Towards multiband optical systems," Proc. Advanced Photonics Congress (Networks), paper NeTu3E.1 (2018).

[10] Winzer, P. J., et al., "Fiber-optic transmission and networking: the previous 20 and the next 20 years [Invited]," Opt. Express 26, 24190-24239 (2018).

[11] Nadal, L., et al., "SDN-enabled S-BVT for SDM Optical Metro/Regional Networks", Proc. ONDM, virtual event (2020).

[12] Nadal, L., et al., "SDN-Enabled S-BVT for Disaggregated Networks: Design, Implementation and Cost Analysis," in Journal of Lightwave Technology, vol. 38, no. 11, pp. 3037-3043 (2020).

[13] Nadal, L., et al., "Meeting the future metro network challenges and requirements by adopting programmable SBVT with direct-detection and PDM functionality," Optical Fiber Technology, Vol. 36, pp. 344-352 (2017).

[14] http://www.passion-project.eu/

[15] Muñoz, R., et al., "The ADRENALINE testbed: An SDN/NFV packet/optical transport network and edge/core cloud platform for end-to-end 5G and IoT services," Proc. EuCNC, doi: 10.1109/EuCNC.2017.7980775 (2017).

[16] Svaluto Moreolo, M., et al., "Experimental Assessment of a Programmable VCSEL-Based Photonic System Architecture over a Multi-Hop Path with 19-Core MCF for Future Agile Tb/s Metro Networks," Proc. OFC 2020, San Diego, CA, USA, (2020).

[17] Gatto, A., Rapisarda, M., Parolari, P., Svaluto Moreolo, M., Neumeyr, C., Boffi, P., "Long-wavelength VCSELbased system exploiting direct DMT modulation and coherent detection for multi-Tb/s metro link", Proc. ONDM, virtual event (2020). 